\title{
Antimicrobial resistance trend of Salmonella typhi and paratyphi from 2011-2013: A descriptive study from tertiary care hospital of Nepal
}

\section{Adhikari $\mathrm{A}^{1}$, Sapkota $\mathrm{S}^{2}$, Bhattarai $\mathrm{U}^{3}$, Raghubanshi $\mathrm{BR}^{4}$}

${ }^{1}$ Arjab Adhikari, MBBS graduate; ${ }^{2}$ Supriya Sapkota, MBBS graduate; ${ }^{3}$ Utsuk Bhattarai, MBBS graduate; ${ }^{4}$ Bijendra Raj Raghubanshi, Lecturer, Departmentof Microbiology, KIST Medical College and Teaching Hospital, Lalitpur, Nepal

\begin{abstract}
Background: Enteric fever is a major public health problem in Nepal. Trends of drug resistance help in choosing optimal empiric therapy.

Objective: The objective of this study was to determine antibiotic resistance pattern of Salmonella enterica serotype typhi and Salmonella enterica serotype paratyphi isolated from blood cultures.

Methodology: A descriptive study was conducted using hospital records at KIST Medical College and Teaching Hospital, Nepal. Isolates from blood cultures between January 2011 and December 2013 were included in this study. Susceptibility to various antimicrobials was determined using modified Kirby-Bauer disk diffusion method. A chi-square for trend analysis was done to evaluate change in susceptibility over three years.

Results: Out of 216 isolates, $68.05 \%$ were Salmonella typhi and 31.48\% were Salmonella paratyphi A. In Salmonella typhi, there was significant increase in cefotaxime resistance $\left(X^{2}=4.951, p<0.05\right)$ and ciprofloxacin resistance $\left(X^{2}=17.506, p<\right.$ $0.001)$ whereas there was significant decrease in ampicillin resistance $\left(X^{2}=4.830, p<0.05\right)$. No resistance was seen against ceftriaxone in Salmonella typhi and Salmonella paratyphi A. Resistance to chloramphenicol and cotrimoxazole was low as well, in both isolates. None of the isolates tested were multidrug resistant.

Conclusion: There is emergence of resistance to cefotaxime whereas resistance to ciprofloxacin has made its use as empiric therapy questionable. Full susceptibility of ceftriaxone makes it an excellent antibiotic for empiric therapy. Decrease in resistance to chloramphenicol, cotrimoxazole and ampicillin may again make these agents useful.
\end{abstract}

Key words: Cefotaxime, Enteric fever, Nepal, Resistance, Salmonella, Typhoid

\section{INTRODUCTION}

Calmonella enterica serotype typhi (ST) cause typhoid $\checkmark$ fever whereas Salmonella enterica serotype paratyphi A (SPA), B (SPB) and C cause paratyphoid fever. Global estimate showed a total of 26.9 million episodes of typhoid fever in 2010, which is comparable to the global estimate in $2000^{1,2}$.

Enteric fever is a major public health problem in Nepal with most cases seen during the monsoon period ${ }^{3}$. In recent years, studies have shown increase in incidence of SPA infection ${ }^{4,5}$. However, ST and SPA were found to cause indistinguishable clinical syndrome ${ }^{6}$.

Address for correspondence

Arjab Adhikari

MBBS graduate from KIST Medical College and Teaching Hospital

E-mail: docarjab@gmail.com
Chloramphenicol became the treatment of choice for enteric fever in $1948^{7}$. In 1973, its resistance became a global problem ${ }^{8}$. Towards the late 1980 s and 1990s, multidrug resistant strains emerged ${ }^{8}$. Multidrug resistance (MDR) is defined as simultaneous resistance to drugs chloramphenicol, ampicillin and cotrimoxazole. Due to the multidrug resistance fluoroquinolones such as ciprofloxacin became the drug of choice for treating enteric fever ${ }^{9,10}$. Several publications have appeared in last decade documenting decreased susceptibility to fluoroquinolones ${ }^{11}$. Understanding of the drug resistance trend helps in choosing the optimal empirical treatment. Thus we conducted a cross-sectional study to understand the antimicrobial resistance pattern in ST and SPA.

\section{METHODOLOGY}

We conducted a descriptive study using hospital records of KIST Medical College and Teaching Hospital, Lalitpur, 
Nepal. Between January 2011 and December 2013, 7063 blood samples were sent to the microbiological laboratory for culture and sensitivity. A total of 216 isolates of ST, SPA and SPB were obtained, all of which were included in this study. Isolates were identified on the basis of colony morphology, staining reaction and biochemical characteristics. Serotype identification was done by using polyvalent and monovalent antisera. Antimicrobial susceptibility test was done using modified Kirby Bauer disk diffusion method as per Clinical and Laboratory Standards Institute guidelines. Susceptibility to antibiotics ampicillin, chloramphenicol, gentamicin, cotrimoxazole, ceftriaxone, cefotaxime and ciprofloxacin were determined. The study was approved by the Institutional Review Board of KIST Medical College and Teaching Hospital.

Statistical analysis was done using IBM SPSS Statistics 20. A chi-square for trend analysis was done to evaluate the change in susceptibility over three years for each serotype. $P$ values of $\leq 0.05$ were used for statistical significance.

\section{RESULTS}

A total of 216 isolates of ST, SPA and SPB were obtained. Among them, $68.05 \%$ were ST and $31.48 \%$ were SPA. Only a single isolate of SPB was obtained over 3 years. Enteric fever was found to be more common in age group 10-19 years (32.9\%) followed by age group 20-29 years (31.5\%) and age group $0-9$ years (16.7\%)(Table 1 ). Males were more commonly affected than females with $54.4 \%$ of ST infection and $66.2 \%$ of SPA infection occurring in males.

Table 2 shows the resistance pattern of ST to various antimicrobials. Increase in resistance to ciprofloxacin and cefotaxime was observed in ST isolates. In 2013, there was a sudden rise in ciprofloxacin resistance $(27.7 \%, \mathrm{n}=65)$ and cefotaxime resistance $(9.4 \%, \mathrm{n}=$ 64). Simultaneous resistance to both ciprofloxacin and cefotaxime were observed in 2 isolates. In contrast, we found gradual decrease in ampicillin resistance over three years. No resistance was noted against ceftriaxone and gentamicin. Resistance to cotrimoxazole (13.3\%, n $=15)$ and chloramphenicol $(13.3 \%, \mathrm{n}=15)$ was low and was only seen in year 2012 .

Table 3 shows the resistance pattern of SPA to various antimicrobials. SPA isolates were fully susceptible to antibiotics ceftriaxone, ciprofloxacin, chloramphenicol and gentamicin. Resistance to ampicillin was seen in $2011(28.6 \%, n=28)$ and $2012(50 \%, n=14)$. SPA isolates were resistant to cotrimoxazole in 2011 (4.8\%, $n=21$ ) only (Table 3). None of the isolates of ST and SPA were multi drug resistant.

Chi square for trend analysis in ST showed linear by linear trends in cefotaxime resistance $\left(X^{2}=4.951, p<\right.$ $0.05)$, ciprofloxacin resistance $\left(X^{2}=17.506, p<0.001\right)$ and ampicillin resistance $\left(X^{2}=4.830, p<0.05\right)$ (Table 2 ). Linear by linear association of antimicrobial resistance was not seen in SPA (Table 3).

Table 1: Age-wise distribution of Salmonella serotypes

\begin{tabular}{|c|c|c|c|c|}
\hline \multirow{2}{*}{ Age Group (years) } & \multicolumn{3}{|c|}{ Isolate } & \multirow{2}{*}{ Total (Percent) } \\
\hline & ST & SPA & SPB & \\
\hline $0-9$ & 26 & 10 & 0 & $36(16.7 \%)$ \\
\hline $10-19$ & 53 & 18 & 0 & $71(32.9 \%)$ \\
\hline $20-29$ & 43 & 25 & 0 & $68(31.5 \%)$ \\
\hline $30-39$ & 8 & 4 & 0 & $12(5.6 \%)$ \\
\hline $40-49$ & 8 & 4 & 0 & $12(5.6 \%)$ \\
\hline $50-59$ & 4 & 3 & 0 & $7 \quad(3.2 \%)$ \\
\hline $60-69$ & 3 & 3 & 0 & $6(2.8 \%)$ \\
\hline $70-79$ & 2 & 1 & 1 & 4 (1.9\%) \\
\hline
\end{tabular}

Abbreviations: ST, Salmonella enterica serotype typhi; SPA, Salmonella enterica serotype paratyphi A; SPB, Salmonella enterica serotype paratyphi B 
Table 2: Antibiotic resistance pattern of Salmonella enterica serotype typhi over three years

\begin{tabular}{|c|c|c|c|c|c|c|c|c|}
\hline \multirow{2}{*}{ Antibiotic } & \multicolumn{2}{|c|}{2011} & \multicolumn{2}{|c|}{2012} & \multicolumn{2}{|c|}{2013} & \multirow{2}{*}{$X^{2}$ for trend } & \multirow{2}{*}{ p-value } \\
\hline & $\% \mathbf{R}$ & Total & $\% \mathbf{R}$ & Total & $\% \mathbf{R}$ & Total & & \\
\hline Cefotaxime & 0 & 43 & 0 & 13 & 9.4 & 64 & 4.951 & 0.026 \\
\hline Ciprofloxacin & 0 & 46 & 0 & 15 & 27.7 & 65 & 17.506 & 0.000 \\
\hline Ceftriaxone & 0 & 40 & 0 & 13 & 0 & 76 & & \\
\hline Chloramphenicol & 0 & 37 & 13.3 & 15 & 0 & 75 & 0.228 & 0.633 \\
\hline Ampicillin & 11.1 & 36 & 22.2 & 9 & 1.4 & 72 & 4.830 & 0.028 \\
\hline Cotrimoxazole & 0 & 45 & 13.3 & 15 & 0 & 74 & 0.112 & 0.738 \\
\hline Gentamicin & 0 & 53 & 0 & 15 & 0 & 71 & & \\
\hline
\end{tabular}

$\mathrm{X}^{2}$ for trend and $\mathrm{p}$ - value could not be calculated for ceftriaxone and gentamicin because there were no changes in resistance over 3 years.

Table 3: Antibiotic resistance pattern of Salmonella enterica serotype paratyphi A over three years

\begin{tabular}{|c|c|c|c|c|c|c|c|c|}
\hline \multirow{2}{*}{ Antibiotic } & \multicolumn{2}{|c|}{2011} & \multicolumn{2}{|c|}{2012} & \multicolumn{2}{|c|}{2013} & \multirow{2}{*}{$X^{2}$ for trend } & \multirow{2}{*}{ p-value } \\
\hline & $\% \mathbf{R}$ & Total & $\% \mathbf{R}$ & Total & $\% \mathbf{R}$ & Total & & \\
\hline Cefotaxime & 0 & 30 & 4.8 & 21 & 0 & 12 & 0.140 & 0.709 \\
\hline Ceftriaxone & 0 & 28 & 0 & 18 & 0 & 14 & & \\
\hline Ciprofloxacin & 0 & 24 & 0 & 21 & 0 & 12 & & \\
\hline Chloramphenicol & 0 & 28 & 0 & 21 & 0 & 14 & & \\
\hline Ampicillin & 28.6 & 28 & 50 & 14 & 0 & 12 & 1.754 & 0.185 \\
\hline Cotrimoxazole & 4.8 & 21 & 0 & 18 & 0 & 13 & 1.136 & 0.286 \\
\hline Gentamicin & 0 & 31 & 0 & 22 & 0 & 12 & & \\
\hline
\end{tabular}

$\mathrm{X}^{2}$ for trend and $\mathrm{p}$ - value could not be calculated for ceftriaxone, ciprofloxacin, chloramphenicol and gentamicin because there were no changes in resistance over 3 years.

\section{DISCUSSION}

Despite increase in incidence of SPA infection ${ }^{4}$ and studies reporting more than $50 \%$ of isolates being SPA $^{12,13}$, we found only $31.48 \%$ of the isolates being SPA. Most of the isolates were ST. Enteric fever caused by SPB is rare. Only a single isolate of SPB was obtained in three years. Enteric fever was most commonly seen in age group 10 - 29 years (Table 1 ). This may be due to high likelihood of this age group to drink unpurified water. The main goal of this research was to determine the resistance of Salmonella enterica serotypes against various antimicrobials.

\section{CIPROFLOXACIN}

Our study suggests that there is a significant rise in ciprofloxacin resistance ( $p<0.001$ ) in ST. None of the isolates tested in 2011 and 2012 were resistant to ciprofloxacin. However, in 2013, 27.7\% $(n=65)$ of the isolates of ST were resistant to ciprofloxacin. In contrast, SPA isolates were sensitive to ciprofloxacin throughout the three years. Increase in ciprofloxacin resistance has also been reported from other studies in Nepal ${ }^{11,14-16}$.
Ciprofloxacin is used as an empiric therapy for enteric fever. Our findings indicate that ciprofloxacin may not be effective in enteric fever. Decrease in sensitivity may be due to overuse of fluoroquinolones and their availability without a prescription in Nepal.

\section{CEFTRIAXONE}

No resistance was seen against ceftriaxone in three years. This demonstrates that ST and SPA are fully susceptible to ceftriaxone. These results are consistent with other studies from Nepal which have shown full susceptibility to ceftriaxone ${ }^{14-16}$. An important implication of these findings is that ceftriaxone is a very good choice for empirical treatment of suspected enteric fever. However, decreased susceptibility has been reported ${ }^{5}$.

\section{CEFOTAXIME}

In contrast to ceftriaxone, which is fully susceptible, resistance to cefotaxime is gradually increasing. An ordered increase in the resistance rate to cefotaxime was observed in ST with $0 \%(n=43)$ in 2011 , and $9.4 \%$ $(n=64)$ in $2013(p=0.026)$. Cefotaxime resistant SPA 
was isolated in 2012. Only one other study, to our knowledge has reported cefotaxime resistant ST and $\mathrm{SPA}^{17}$. Unfortunately, it did not mention the number or percentage of isolates resistant to cefotaxime. However, the resistant isolates were found to be extended spectrum beta-lactamase (ESBL) test positive. Moderate susceptibility to cefotaxime (not resistance) has been reported by Pokharel et al. ${ }^{13}$. They found three isolates of ESBL producing SPA. In contrast to these studies, full susceptibility of isolates to cefotaxime has been documented by Shrestha et al. ${ }^{16}$. As ESBL gene is mobile in nature, the mechanism of resistance in ST and SPA may be due to horizontal transfer of ESBL gene. Future studies should look for presence of ESBL gene if cefotaxime resistant ST or SPA is isolated.

\section{CHLORAMPHENICOL, COTRIMOXAZOLE, AMPICILLIN}

Our study indicates that there has been a significant decrease in ampicillin resistance in ST ( $p=0.028)$. Resistance to cotrimoxazole (13.3\%, $\mathrm{n}=15)$ and chloramphenicol $(13.3 \%, \mathrm{n}=15)$ was low as well and seen only in 2012.

In SPA isolates, no resistance was observed against chloramphenicol. However, ampicillin resistance was seen in 2011 (28.6\%, n = 28) and 2012 (50\%, n =14). Cotrimoxazole resistance was seen only in 2011 (4.8\%, $\mathrm{n}=21$ ).

Decrease in resistance in ST and SPA may be due to decrease in use of these traditional first line antimicrobials. A significant decrease in resistance to chloramphenicol and cotrimoxazole has also been reported from Nepal ${ }^{11,16,18}$. Decrease in resistance may again make these antibiotics useful in near future.

\section{REFERENCES}

1. Crump JA, Luby SP, Mintz ED. The global burden of typhoid fever. Bull World Health Organ. 2004 May;82(5):346-53.

2. Buckle GC, Walker CLF, Black RE. Typhoid fever and paratyphoid fever: Systematic review to estimate global morbidity and mortality for 2010. J Glob Health. 2012 Jun;2(1):10401.

3. Karkey A, Aryjal A, Basnyat B, Baker S. Kathmandu, Nepal: still an enteric fever capital of the world. J Infect Dev Ctries. 2008;2(6):461-5.

4. Ochiai RL, Wang $X$, von Seidlein L, Yang J, Bhutta ZA, Bhattacharya SK, et al. Salmonella paratyphi A rates, Asia. Emerg Infect Dis. 2005 Nov;11(11):1764-6.
None of the isolates tested were MDR, which is defined as simultaneous resistance to chloramphenicol, ampicillin and cotrimoxazole. This shows that MDR strains are decreasing. This finding is consistent with decreasing trend of MDR strains in Nepal' ${ }^{11}$. Decrease in MDR may again be attributed to decrease in use of traditional first line drugs.

\section{GENTAMICIN}

No resistance was seen against gentamicin. Other studies have also reported similar finding ${ }^{14,15}$. However, because of toxicity its use is limited.

There were some limitations of our study. Minimal inhibitory concentrations of the antibiotics were not determined. Isolates were not tested against nalidixic acid, which is considered as a maker for decreased sensitivity to fluoroquinolones ${ }^{19}$. As this study was conducted using hospital records, not all the isolates were tested against all the antibiotics used for this study.

\section{CONCLUSIONS}

In conclusion, there is emergence of resistance tocefotaxime. Significant resistance to ciprofloxacin makes its use questionable as an empiric therapy. However, ceftriaxone is fully susceptible which makes it a useful empirical antibiotic. Decrease in resistance to chloramphenicol, cotrimoxazole and ampicillin may again make these agents a therapeutic option.

\section{ACKNOWLEDGEMENT}

We would like to thank Prof. Dr. Amita Pradhan, KIST Medical College and Teaching hospital for helping us in methodology and statistical analysis. We would also like to thank Dr. Bhusan Raj Timilsina and Dr. Aman Dutta for their support during the research period.

5. Raza S, Tamrakar R, Bhatt CP, Joshi SK. Antimicrobial susceptibility patterns of Salmonella typhi and Salmonella paratyphi A in a tertiary care hospital. J Nepal Health Res Counc. 2012 Sep;10(22):214-7.

6. Maskey AP, Day JN, Phung QT, Thwaites GE, Campbell Jl, Zimmerman M, et al. Salmonella enterica serovar Paratyphi A and S. enterica serovar Typhi cause indistinguishable clinical syndromes in Kathmandu, Nepal. Clin Infect Dis. 2006 May 1;42(9):1247-53.

7. Mirza SH, Beechmg NJ, Hart CA. Multi-drug resistant typhoid: a global problem. J Med Microbiol. 1996;44(5):317-319. 
8. Parry CM, Hien TT, Dougan G, White NJ, Farrar JJ. Typhoid Fever. N Engl J Med. 2002 Nov 28;347(22):1770-82.

9. Bhutta ZA. Current concepts in the diagnosis and treatment of typhoid fever. BMJ. 2006 Jul 8;333(7558):78-82.

10. Bhan MK, Bahl R, Bhatnagar S. Typhoid and paratyphoid fever. Lancet Lond Engl. 2005 Sep 27;366(9487):749-62.

11. Karki S, Shakya P, Cheng AC, Dumre SP, Leder K. Trends of etiology and drug resistance in enteric fever in the last two decades in Nepal: a systematic review and meta-analysis. Clin Infect Dis. 2013 Nov;57(10):e167-176.

12. Pokharel P, Rai SK, Karki G, Katuwal A, Vitrakoti R, Shrestha SK. Study of enteric fever and antibiogram of Salmonella isolates at a teaching hospital in Kathmandu Valley. Nepal Med Coll J. 2009 Sep;11(3):176-8.

13. Pokharel BM, Koirala J, Dahal RK, Mishra SK, Khadga PK, Tuladhar NR. Multidrug-resistant and extendedspectrum beta-lactamase (ESBL)-producing Salmonella enterica (serotypes Typhi and Paratyphi A) from blood isolates in Nepal: surveillance of resistance and a search for newer alternatives. Int J Infect Dis. 2006 Nov;10(6):434-8.

14. Singh SD, Shrestha S, Shrestha N, Manandhar S. Enteric fever in Children at Dhulikhel Hospital. J Nepal Paediatr Soc [Internet]. 2013 Feb 23 [cited
2016 Aug 20];32(3). Available from: http://nepjol. info/index.php/JNPS/article/view/6682

15. Acharya D, Bhatta DR, Malla S, Dumre SP, Adhikari N, Kandel BP. Salmonella enterica serovar Paratyphi A: an emerging cause of febrile illness in Nepal. Nepal Med Coll J. 2011 Jun;13(2):69-73.

16. Shrestha KL, Pant ND, Bhandari R, Khatri S, Shrestha B, Lekhak B. Re-emergence of the susceptibility of the Salmonella spp. isolated from blood samples to conventional first line antibiotics. Antimicrob Resist Infect Control [Internet]. 2016 May 25 [cited 2016 Aug 20];5. Available from: http://www.ncbi.nlm.nih. gov/pmc/articles/PMC4881163/

17. Gautam K, Pokhrel BM, Bhatta DR, Shrestha CD. Studies on extended spectrum beta lactamase (ESBL) producing salmonella isolates from clinical samples of Nepal. Nepal Med Coll J NMCJ. 2012 Sep;14(3):204-6.

18. Chand HJ, Rijal KR, Neupane B, Sharma VK, Jha B. Re-emergence of susceptibility to conventional first line drugs in Salmonella isolates from enteric fever patients in Nepal. J Infect Dev Ctries. 2014 Nov;8(11):1483-7.

19. Hakanen A, Kotilainen P, Jalava J, Siitonen A, Huovinen P. Detection of Decreased Fluoroquinolone Susceptibility in Salmonellas and Validation of Nalidixic Acid Screening Test. J Clin Microbiol. 1999 Nov;37(11):3572-7. 